Thorax (1965), 20, 1.

\title{
Neonates and chest surgery
}

\author{
H. E. BE L L \\ From the Newcastle Region Thoracic Surgical Unit, Seaham Hall Hospital, Co. Durham
}

The theme of this article is the management of thoracotomy in the neonate in respect of anaesthesia, ventilation, blood replacement, and postoperative care.

The principles of management of a chest operation in the newborn are much the same as during other periods of life, but the margin of safety in the neonate is small; many of these babies are premature and many have other congenital abnormalities.

\section{ANAESTHESIA AND VENTILATION}

I believe that, of the various techniques available, light cyclopropane anaesthesia is the least likely to jeopardize survival. I use manual ventilation throughout the operation.

The anaesthetic equipment is simple, but to avoid trouble during the operation the various components must be chosen with care. Odd bits gathered together just before operation will not do. A new plain rubber Magill tube is used for each case and checked for patency beforehand. A stock of tubes, $2 \cdot 5,3$, and $4 \mathrm{~mm}$. in diameter, are kept (those made by J. G. Franklin \& Sons Ltd. are suitable). The appropriate length of tube for a particular baby is found by measuring the distance between the suprasternal notch and the mouth, following the curve of the air passages. A $3 \mathrm{~mm}$. diameter tube is the appropriate size for most neonates, but sometimes a final decision as to the right size can only be made after intubation has been attempted. Any attempt to screw home too big a tube is likely to result in laryngeal oedema, whereas a troublesome gas leak will accompany controlled ventilation with a tube that is too small. The endotracheal tube is attached to a miniature Magill (right-angled with port for suction) connexion and bound on with a few turns of adhesive strapping; this prevents splitting of the tube and separation from the connexion. A right-angled connexion is chosen because a suction catheter can be passed quickly and easily through it, and it can easily be anchored in stable position at the mouth. A clear airway is better established and maintained by this method than by any other. Any objections on the score of turbulent gas flow and a consequent increase of respiratory work are of little significance because, during controlled respiration, the respiratory work is done by the anaesthetist. The endotracheal connexion is joined in succession to a short, loose-fitting catheter mount, an Ingle valve, a $3 \mathrm{oz}$. (85 g.) soda-lime canister and a 0.5 litre reservoir bag.

Atropine, gr. 1/800 (0.08 mg.) s.c.i., is given half an hour before operation with the intention of reducing bronchial secretions. Since cyclopropane anaesthesia is used, atropine is probably unnecessary ; nevertheless, one should not lightly dispense with any measure that may contribute to a clear airway in a small baby. Atropine is conventionally given before cyclopropane anaesthesia to prevent vagal slowing of the heart. I do not know of any evidence to establish that a neonate under anaesthesia is better off with a fast heart rate, and I would not prescribe atropine except to reduce secretion. Cyclopropane does not cause cardiac irregularities in children (Shannon, D. W., 1950, personal communication).

When all is ready in the theatre the baby is brought along in his incubator, removed and placed in warm towelling on the table. If cyanosis is evident oxygen is given before intubation. No particular position of the head or neck seems to have any advantage over the horizontal in so far as intubation of the newborn is concerned; but gentle pressure on the larynx from the outside may help one to see the laryngeal opening. A straight-bladed Magill laryngoscope, slightly lubricated, is used, and before the endotracheal tube is inserted a bacteriological swab of the pharyngeal secretions is taken. The endotracheal tube should not be daubed with lubricant; the slightest smear is enough.

Most authorities now recommend intubation before an anaesthetic or relaxant is given ; I think this is sound advice. With the tube in position, from the start the baby is spared any period of precarious ventilation control; bronchial secretions can be cleared out straight away, and 
abnormalities of the larynx discovered in favourable circumstances. In one baby with oesophageal atresia the larynx and upper parts of the trachea and oesophagus were one common passage. A large endotracheal tube pushed into 'the oesophagus' provided good ventilation, but the defect was irreparable.

After intubation the baby is quickly put to sleep with $50 \%$ cyclopropane in oxygen. When body movements cease and the breathing becomes regular the concentration of cyclopropane is cut to 5 to $10 \%$ and manual inflation is begun. The total gas flow necessary depends on the amount of laryngeal leak but in any case must not be less than $1,000 \mathrm{ml}$./min. (e.g., $900 \mathrm{ml}$. $\mathrm{O}_{2}, 100 \mathrm{ml}$. $\mathrm{C}_{3} \mathrm{H}_{6}$ ). A troublesome leak can be reduced by the pressure of a finger on each side of the larynx with the head and neck slightly extended. Packing of the pharynx is not effective as a seal. Suitable fullness of the reservoir bag is achieved by balancing the total flow rate, leaks, and expiratory valve setting.

The baby is usually turned on to his side, the head and the soda-lime canister resting on a white towel ; this makes assessment of the colour easier. The reservoir bag should protrude over the top end of the table for ease of handling. A piece of Cramer wire inserted under the table covering and suitably bent serves as an anaesthetic screen; the sterile towel placed over this should be white. A good light beamed on to the baby's face completes the arrangements. Everything must be in order before the operation is begun; faults are hard to correct later.

Anaesthesia for neonatal chest surgery should provide sleep, a still baby, and good ventilation. Deep anaesthesia is not necessary to fulfil these conditions. Neonates willingly surrender their respiration to intermittent positive pressure (Rees, 1958), and anaesthesia deep enough to prevent body movement will provide sleep. The aim is to keep the baby at a depth that prevents movement, and no more. At this depth muscular tone is present in the limbs, and this is a valuable guide ; relaxed flaccid limbs mean that the anaesthesia is deeper than need be. (I have cyclopropane solely in mind here, and not, of course, the use of relaxants.)

Anaesthesia is established with $50 \%$ cyclopropane, after which manual ventilation with $10 \%$ cyclopropane is begun. Subsequent management is determined by the response of the baby: most will gradually become lighter on $10 \%$ cyclopropane, as shown by increasing muscle tone and eventually movements. (The presence and degree of muscle tone is most easily assessed by moving a hand to and from the shoulder.) The anaesthesia is thereupon deepened by inflation with $50 \%$ cyclopropane until muscle tone disappears, when a return to $10 \%$ cyclopropane is made. This cycle is repeated, when indicated, throughout the operation. Small babies in poor general condition are likely to remain immobile and flaccid on $10 \%$ cyclopropane: in this case the concentration should be reduced to $5 \%$, with deepening as before by $50 \%$ cyclopropane as occasion demands. It is best to keep to the concentrations of cyclopropane mentioned $(50 \%$ and $10 \%$ or $5 \%$ ), a variety of concentrations resulting in confusion and erratic anaesthesia.

Cyclopropane used as described above is more satisfactory than any other form of anaesthesia for neonatal chest surgery. It is, however, explosive, which precludes the use of diathermy; this is unfortunate as light cyclopropane anaesthesia results in more bleeding, from the chest wall in particular, than other anaesthetics. The use of relaxant drugs with nitrous oxide anaesthesia in a newborn baby, for the main purpose of allowing diathermy, is based on questionable priorities. Relaxant drugs are not predictable in their effects on the newborn; respiration at the end of the operation, even in a wide awake baby, is often critical enough without any lag effect from a relaxant. Since I believe a blood transfusion is always necessary to maintain blood volume, the extra loss brought about by cyclopropane can be made good by a small transfusion.

The lungs are ventilated by rhythmic manual compression of the reservoir bag. The rate should be slightly higher than the physiological norm, and I think the tidal volume is usually increased as well. Whether it is the result of cyclopropane, hyperventilation, the imposition of a conflicting rhythm, or more likely a combination of all three, control of respiration is easily maintained. A high rate of inflation must be avoided because it will probably be accompanied by inadequate expiration and deterioration of the general clinical condition (Engström, Herzog, Norlander, and Swensson, 1962). The reservoir bag must be of small capacity $(0.5$ litre) or it will not be possible to appreciate the small volume change and flow sensation indicative of effective inflation. Resistance to inflation is due to respiratory obstruction or lung compression by the primary pathological condition. Obstruction of an endotracheal tube by kinking could be obviated by the use of an armoured tube, but these have disadvantages, and 
kinking has not occurred with the arrangements described here.

The other causes of obstruction are mucus, mucopus, radio-opaque material ${ }^{1}$ or blood in the air passages ; bronchospasm is rare. Some degree of bronchitis is invariably present in babies who have a tracheo-oesophageal fistula ; it is caused by the spill of digestive secretions into the trachea through the fistula. The presence of secretions and mucopus can be heard (rattling respiration) before obstruction becomes evident ; but this may not be so. When in doubt aspirate. Fresh blood in the air passages of a small baby is dangerous: it is prone to solidify at the end of the endotracheal tube and to produce obstruction that cannot be removed. From the first sign of blood, aspiration must continue until it has been cleared; $0.5 \mathrm{ml}$. normal saline or dilute sodium bicarbonate injected down the endotracheal tube may help. A haematoma of the lung caused by retraction is the commonest cause of blood in the bronchi in these cases.

In conditions of lung compression ventilation may be a problem. The tidal volume may be so small as to be imperceptible on bag compression. In such a situation the temptation to use a high inflation pressure must be resisted. High inflation pressure contributes little improvement to ventilation when the basic fault is restricted lung volume and carries the risk of lung rupture. An ear placed on the bag may enable one to hear gas movement to and from the lungs, and, provided that cyanosis does not appear, things are probably not too bad. At any rate one can hope for an improvement once the chest has been opened (though in the case of a large gastrogenous cyst arising from the upper mediastinum improvement had to await aspiration of the cyst fluid). Lung compression by the primary condition may not be responsible for inflation difficulty, and in these cases one should be alert for a coexistent respiratory obstruction; the free passage of a catheter, followed by aspiration, will establish that no obstruction exists. A rubber Jaques catheter, No. 6-7 F.G., can easily be passed down the endotracheal tube for suction purposes, and it is soft enough not to damage the bronchial mucosa, an important point when aspiration has to be repeated. The end containing the lateral hole should first be cut off so as to provide an end hole, and the edge should be

\footnotetext{
1 The malpractice of diagnosing oesophageal atresia by radioopaque swallow has not yet been eradicated. Some of the radioopaque medium invariably enters the lungs, with detrimental effect. A diagnosis of oesophageal atresia is made when a size 12 F.G. Jaques catheter, passed down the oesophagus, is halted at a distance of $4-4 \frac{1}{2}$ in. $(10-11.25 \mathrm{~cm}$.) from the mouth: no further proof is needed.
}

trimmed. If this is not done the lateral opening sticks to the inner wall of the endotracheal tube and to the mucosa, making introduction difficult and suction ineffective.

There is no place for mechanical ventilators. The primary concern of the anaesthetist is to provide adequate ventilation, and to this end he should devote his attention. To attach a baby to a ventilator 'so that the anaesthetist is free to do other jobs' is dangerous.

A Malecot catheter is inserted by the surgeon into the pleural cavity before closure of the chest and joined by narrow tubing to a water seal drainage bottle. The tubing should be of appropriate bore for a neonate, and this applies particularly to the glass part of the tube (inside the drainage bottle), wherein a column of water swings up and down on respiration. The height of the swing is irrespective (within reasonable limits) of the bore of the tube, but the volume of water represented by the swing is greater in a large tube than in a small one. This volume is dead space over and above physiological dead space. Taking physiological dead space in a baby as $1 \mathrm{ml}$. per lb. body weight (Radford, 1955), the use of a large tube could double the total dead space, with consequent dyspnoea. (There is also the extra respiratory work involved in raising a large weight of water.)

Five minutes before the end of the operation $100 \%$ oxygen is given, and spontaneous respiration is encouraged. When the baby is breathing and moving vigorously, the bronchial tree and pharynx are aspirated and the endotracheal tute is removed after a full inspiration.

\section{BLOOD TRANSFUSION}

To maintain a baby in the best condition for survival after a chest operation, blood lost at operation should be replaced by transfusion. The guiding principle of blood replacement in infants is that a slight over-transfusion is less harmful than under-transfusion. Blood loss estimates usually err on the low side. I do not think that the loss of blood during thoracotomy in a neonate is ever so small that transfusion can be omitted. Potts (1959) has stated that a $5 \mathrm{lb}$. infant may not need more than $50 \mathrm{ml}$. of blood during operation, but that amount is the difference between success and failure. Rickham (1957) maintains that blood lost during operation should be replaced, and that while over-infusion is harmful, he has seen no ill-effects from slight over-transfusion during operation. Despite defects, I find the weighing of swabs useful as a coarse indication 
of the loss of blood and in deciding on the amount of blood to be given. The estimate from weighing swabs, general condition, body weight, and tendency to ooze are all taken into consideration. As a rough guide, the old transfusion formula for babies, 10 to $15 \mathrm{ml}$. per lb. body weight for an operation of moderate severity, fits well with the other estimates.

Since the major part of the loss of blood occurs during opening of the chest it is a good plan to aim at getting 20 to $30 \mathrm{ml}$. blood into the baby by the time the operation begins. Pre-loading with $30 \mathrm{ml}$. does no harm and effectively cushions subsequent losses; it is an insurance against the effects of drip failure should this occur early in the operation. The supervision of the drip should be delegated to some experienced person: it is not satisfactory for the anaesthetist to have this to do as a sideline. The blood must enter the baby's circulation and not escape through loose connexions under the operation towelling.

The best site for the drip is the umbilical vein and, when possible, this is used. Owing to the large size of this vein the drip runs freely and too much blood must not be run in. When the baby is more than 4 to 5 days old, the umbilical vein cannot be used, and the saphenous vein at the ankle is cannulated under local anaesthesia. In distinction to the umbilical vein, drips here tend to be sluggish, though an improvement in rate can be brought about by injecting 0.5 to $1 \mathrm{mg}$. pethidine in $1 \mathrm{ml}$. saline directly into the drip near the ankle. This small amount of pethidine does the baby no detectable harm, and I do not know of any other substance that has such a beneficial effect on a slow drip. A scalp vein drip is not satisfactory; it is precarious and slow, and, though the rate of transfusion can be boosted further by incorporating a tap and syringe in the drip line, considerable haemolysis results from squirting blood through small needles. In an emergency, blood can be injected directly into the aorta or superior vena cava by the surgeon.

There are advantages in using freshly drawn heparinized blood for neonatal surgery (Carter, Waterston, and Aberdeen, 1962).

Good ventilation, light anaesthesia, and replacement of blood lost are the fundamentals of management of thoracic surgery in the newborn. If the general clinical condition deteriorates, little can be done apart from giving $100 \%$ oxygen and attending to blood balance. A guide to the general clinical condition I find useful is this: baby pink and pulses palpable - condition good; baby pale, pulses palpable or baby pink, pulses not palpable -condition satisfactory, but check ventilation and blood balance; baby pale, pulses not palpablecondition serious. I do not think that cardiac stethoscopes (the heart action can be seen in thoracic operations), sphygmomanometers, pulse monitors or E.C.G. tracings contribute enough additional information about a baby's condition to merit their use ; they may be distracting.

\section{TEMPERATURE}

An effort should be made to prevent the temperature of these babies from falling during operation. Cold predisposes to sclerema in premature babies and has a depressant effect that may result in apnoea (Bush and Stead, 1962). The factors responsible for a fall in temperature are the exposure of a large area of open chest, reduced heat production, and the transfusion of cold blood.

The large loss of heat from an open pleural cavity was remarked on by Wright (1952), and chest operations are long. To combat this loss the theatre temperature should be high - certainly not less than $75^{\circ} \mathrm{F}$.- and ideally the humidity should also be high to reduce evaporation from moist surfaces (latent heat of vaporization). To counteract losses, some heat may be contributed to the baby by laying it on a heated blanket or some other device ; care must be taken to avoid burns ; the blanket temperature must not exceed $42^{\circ} \mathrm{C}$.

One of the advantages of light cyclopropane anaesthesia is the preservation of some muscle tone and consequently of heat production. Blood for transfusion should be warmed to a temperature approaching that of the body before being given.

The effective remedy for a steadily falling temperature is to get the operation finished without delay and return the baby to a warm incubator.

\section{POST-OPERATIVE CARE}

Respiratory complications are the main cause of death (DeBoer and Potts, 1957): post-operative care centres on the prevention of these. The baby is nursed in a warm, humid, bacterially clean atmosphere under antibiotic cover. Survival depends on nursing care. The standard of this care will be higher if the nursing staff understands the treatment.

Warmth and humidity are closely connected: a warm atmosphere must be moist or the respiratory secretions dry and obstruct the airway, 
with ultimate infection if not immediate asphyxia. Respiratory secretions are increased after thoracotomy and this excess must be removed. Coughing is the normal mechanism of removal from the major bronchi, but the cough reflex is weak in the newborn and may be non-existent when the baby has undergone a chest operation (DeBoer and Potts, 1957). Crying also clears the bronchial tree, and if the secretions can thereby be expressed as far as the pharynx by the baby they can be aspirated by suction; at the same time an excess of saliva can also be aspirated. Disposal of saliva is a special problem in patients with oesophageal atresia: although swallowing is made possible by operation it is nevertheless defective. At the site of anastomosis the oesophagus is narrow, and peristalsis is absent for some distance above and below; if saliva is not regularly removed by suction there is a risk that it will be inhaled.

In summary, the baby is stimulated to cryby squeezing the tendo Achillis-every quarter hour, and secretions, both salivary and bronchial, are cleared from the pharynx by suction. To be effective, the rubber Jaques catheter used for the purpose must be inserted as far as the larynx, but no further for fear of damaging the anastomosis ; the catheter is marked at the appropriate distance from the tip and inserted as far as this mark.

The baby is turned on to his other side at quarter-hourly intervals to counteract gravitational pooling of secretions. It is both convenient and desirable for the suck-out of the pharynx to be done just before the turning. If, as time goes on, the secretions are scanty, the intervals between turning may be lengthened. If at any time bubbling respiration persists and the secretions cannot be got rid of by oro-pharyngeal suction, direct tracheo-bronchial suction will be needed (q.v.).

An additional benefit from a humid atmosphere is the decreased insensible loss of water. This not only minimizes the need for parenteral fluids but reduces loss of heat (latent heat of vaporization).

The oxygen content of the atmosphere in the incubator is kept at $30 \%$ to $35 \%$ until the baby is over the immediate effects of operation and the colour is pink without oxygen enrichment. In the absence of cyanosis this concentration of oxygen should not be exceeded. Apart from the danger of retrolental fibroplasia, a high concentration of oxygen has a depressant effect on respiratory movement with an increased danger of lung collapse. (The activity of the neonatal respiratory centre is governed more by $\mathrm{O}_{2}$ than $\mathrm{CO}_{2}$ concentration) (Rutten, 1962). If cyanosis appears, there should be no hesitation in using as high an oxygen concentration as is necessary to prevent it. If used in this way there is no danger of oxygen causing retrolental fibroplasia.

The principle of water-seal drainage of the chest should be explained to all concerned with aftercare. To be effective, the drainage tube must remain in free communication with the pleural cavity until air leaks have ceased and the lung has fully expanded. Free communication is shown by the respiratory swing of the water column in the tube at the bottle end. This respiratory swing decreases progressively in size and disappears when the tube inlet becomes covered by expanded lung and sealed off from the rest of the pleural cavity by exudate; this is usually complete by 24 hours after operation, when the tube is removed. A big respiratory swing indicates incomplete lung expansion and pneumothorax; the baby should be made to cry, so as to expand the lung and blow off the pneumothorax. No swing means an expanded lung or a blocked tube. The danger of a blocked tube is tension pneumothorax, but provided that there is no evidence of respiratory distress the absence of swing means that the tube is ready for removal.

The earliest sign of respiratory distress is jerky breathing. Normally an infant's respiratory excursions are smooth and the colour is pink; cyanosis or jerky breathing means that complications are developing.

Physical methods of examination are of limited use in post-operative care. Auscultation reveals the presence or absence of air entry and adventitious sounds, and is supporting evidence for the condition of the lungs. (Auscultation is made easier by the use of Nobecutane instead of gauze and adhesive strapping as a wound dressing.) But for the positive establishment of the normal, or the elucidation of complications, a chest radiograph is essential. Accordingly, a chest radiograph is taken on each of the first three post-operative mornings and is repeated whenever there is doubt about the presence of complications. All films are taken with the baby held upright ; thus effusions are apparent and are seen as soon as they have developed so that action can be taken without delay.

The main complications are pneumothorax or aspiration collapse-pneumonia. The reappearance of pneumothorax after the water seal drain has been removed is sinister after the repair of oesophageal atresia. It may mean that the anasto- 
mosis has broken down. Whatever the reason, water-seal drainage must be reinstated. Atelectasis calls for aspiration of the trachea and bronchi as soon as possible. This may be done by bronchoscopy, but I prefer to pass an endotracheal tube and through it a suction catheter. This is less shocking to the baby and less damaging to the mucosa than bronchoscopy, a feature of some significance when sucking out has to be repeated. A recent successful case of oesophageal atresia had to be sucked out 12 times within a week. The main disadvantage is that the right or left bronchus cannot be selectively entered, but since the right lung is the commoner source of trouble (oesophageal atresia being dealt with by right thoracotomy) and can more easily be entered blindly, this matter is of little moment. In the adult, the angle that the right bronchus makes with the trachea is less than that made by the left bronchus, hence a suction catheter introduced blindly enters the right bronchus. Smith (1959) stated that in infants these angles are equal (both $50^{\circ}$ ) so that the chances of the catheter entering either side will also be equal. Be this as it may, when collapse occurs on the right side the mediastinum moves across to the right, bringing the trachea more in line with the right bronchus. If suction by a catheter introduced blindly fails to produce a clear radiograph, recourse must be had to bronchoscopy. Bronchoscopy should not be undertaken lightly in a neonate. Gentleness is imperative; contusion of the bronchial mucosa will be followed by swelling and exudate, producing further obstruction and in turn necessitating a repeat bronchoscopy; a vicious circle is thus entered and the baby can be 'bronchoscoped to death'. The arrangements for bronchoscopy are the same as for a suck-out by endotracheal tube and catheter. It is important to have facilities ready for inflating the lungs with oxygen via the bronchoscope. A large endotracheal tube inserted into the end of the bronchoscope and connected up to a reservoir bag and oxygen supply makes a suitable appliance.

Attendants who have a cold must avoid contact with the baby. Disposal of respiratory tract secretions is a serious problem in these babies in normal convalescence; the additional infected secretion of the common cold may prove overwhelming.

The air supply to the incubator should be passed through a bacteria filter or led in by a duct from the outside atmosphere. As a bulwark against infection, the antibiotic therapy most likely to be effective against the ambient hospital bacteria is given from the start: this applies $\overrightarrow{\vec{F}}$ particularly to patients with oesophageal atresiain whom infection is likely to be quickly $\frac{C}{0}$ disastrous.

The avoidance of chest complications is in $\vec{\nabla}$ conflict with the need for feeding after the repair of the oesophageal atresia. Repair of oesophageal ${ }^{\text {os }}$ atresia does not equip the baby with a normal $\overrightarrow{0}$ oesophagus: the lumen is at first narrow at the $\overrightarrow{-}$ site of anastomosis, and peristalsis is absent for $\vec{\sigma}$ some distance above and below. Swallowing is $\overrightarrow{\overrightarrow{2}}$ consequently slow, with the risk of overflow of $\vec{x}$ the feed into the lungs. This problem is most acute? for the first week after operation; thereafter:oedema of the suture line subsides, the lumen $\vec{\circ}$ of the oesophagus increases in size at this level, and an improvement in swallowing can be $\vec{c}$ expected. Early feeding of premature babies is now recommended so as to avoid the dangers of neonatal hypoglycaemia-apnoeic attacks, con-₹ vulsions, permanent brain damage, and death. It $\overrightarrow{0}$ was thought that the use of a small calibre naso- $\mathrm{G}$ gastric tube $(1 \mathrm{~mm}$. external diameter) would be the solution to feeding difficulties in the post-O operative phase of oesophageal atresia: the baby could be fed easily without risk of food aspiration and with minimal swallowing strain on the anasto- $\frac{2}{D}$ mosis; and the small calibre of the tube would $\stackrel{\varrho}{\Rightarrow}$ not block the lumen of the oesophagus for saliva.음 But our experiences have been disappointing. The presence of the tube stimulates production of mucus and swallowing (Rutten, 1962), and, despite its small calibre, the tube obstructs the oesophagus-for sticky mucus in particular-at the level of anastomosis. The danger of bronchial ${ }^{\times}$ infection from the aspiration of mucus is 3 increased. In addition, stagnant mucus within the oesophagus favours infection at the suture line; 을 another possibility is that the ascent of gastric juices to the suture line is facilitated (capillary flow? between the outside of the tube and the oeso- $\frac{}{0}$ phageal mucosa). The net result of tube feeding is that although feeding itself is easy, the chances $N$ of breakdown of the anastomosis through mechanical, infective, or chemical processes areㅇ increased by the presence of the tube, and there ${ }^{\omega}$ is a greater risk of aspiration of mucus and its? consequences. An important contribution to the solution of the feeding problem was made by Potts (1962); he stated that the baby is going to swallow anyway, and he might just as well swallow a little clear water and keep his oesophagus clean,,$\vec{D}$ thus aiding the passage of swallowed mucus and? preventing stagnation and the risk of infection ato the site of anastomosis. If the anastomosis is 
watertight at the time of operation, the oesophagus is fit for use straight away and is probably the better for being used. If the oesophagus is not watertight after anastomosis, the chances of its becoming so later are negligible and are not improved by withholding oral feeding. Should one hold opposite views, then to be logical oral feeding must be withheld for over one week. (Rutten (1962) states that the critical period for the anastomosis lies between the fifth and eighth days after operation.) To maintain a satisfactory fluid balance during this period by parenteral means is not easy. Gastrostomy also has its difficulties.

In summary, the indications are that early oral feeding offers the baby the best prospect for survival. For those interested in the detailed technique of oral feeding after repair of oesophageal atresia, I can do no better than refer them to an article by DeBoer and Potts (1957).
I should like to thank my colleagues Mr. S. G. Griffin, Mr. R. Dobson, Dr. J. B. Heycock, and Dr. T. C. Noble.

\section{REFERENCES}

Bush, G. H., and Stead, A. L. (1962). The use of D-tubocurarine in neonatal anaesthesia. Brit. J. Anaesth., 34, 721.

Carter, R. E. Bonham, Waterston, D. J., and Aberdeen, E. (1962). Hernia and eventration of the diaphragm in childhood. Lancet, $1,656$.

DeBoer, A., and Potts, W. J. (1957). Congenital atresia of the esophagus with tracheo-esophageal fistula. Surg. Gynec. Obstet., 104, 475.

Engström, C.-G., Herzog, P., Norlander, O. P., and Swensson, S. A. (1962). Ventilation nomogram for the newborn and small children to be used with the Engström respirator. Acta anaesth. scand., 6, 175.

Potts, W. J.'(1959). The Surgeon and the Child, p. 24. Saunders, Philadelphia.

Philadelphia.
(1962). Quoted by Rutten (1962).

Radford, jun., E. P. (1955). Ventilation standards for use in artificial respiration. J. appl. Physiol., 7, 451.

Rees, G. J. (1958). Neonatal anaesthesia. Brit. met. Bull., 14, 38.

Rickham, P. P. (1957). The Metabolic Response to Neonatal Surgery,

p. 77. Harvard University rress, Cambridge, Mass.
Rutten, A. P. M. (1962). Oesophageal Atresia, p. 106. Koersen en Zonen, Amsterdam.

Smith, R. M. (1959). Anesthesia for Infants and Children, p. 28. Mosby, St. Louis.

Wright, S. (1952). Applied Physiology, 9th ed., p. 369. Oxford University Press, London. 